\title{
Níveis de lisina em rações para frangos de corte determinados com base em uma abordagem econômica
}

\author{
Jefferson Costa de Siqueira ${ }^{1,2}$, Nilva Kazue Sakomura ${ }^{3}$, Juliano César de Paula Dorigam ${ }^{1}$, \\ Gabriela Geraldi Mendonça ${ }^{4}$, Fernando Guilherme Perazzo Costa ${ }^{5}$, João Batista Kochenborger \\ Fernandes $^{6}$, Leilane Rocha Barros Dourado ${ }^{7}$, Dáphinne Cardoso Nagib do Nascimento ${ }^{1}$
}

\footnotetext{
1 Pós-graduação em Zootecnia/FCAVIUNESP, Jaboticabal, SP.

2 Centro de Ciências Agrárias e Ambientais/Universidade Federal do Maranhão (CCAA/UFMA), Chapadinha, MA.

${ }^{3}$ Departamento de Zootecnia/FCAVIUNESP. Jaboticabal, SP. Bolsista CNPO e membro INCT.

${ }^{4}$ Graduando em Zootecnia/FCAV/UNESP, Jaboticabal, SP.

5 Departamento de Zootecnia/Universidade Federal da Paraíba (UFPB), Areia, PB.

${ }^{6}$ Centro de Aqüicultura da Universidade Estadual Paulista (CAUNESP), Jaboticabal, SP.

${ }^{7}$ Departamento de Zootecnia/Universidade Federal do Piauí (UFPI), Bom Jesus, PI.
}

RESUMO - O objetivo neste estudo foi estimar os níveis ótimos de lisina digestível em rações para frangos de corte em crescimento (22 a 35 dias) e terminação (35 a 42 dias) com base na análise econômica da alimentação. Foram conduzidos dois experimentos, cada um com 600 frangos de corte machos da linhagem Cobb 500 distribuídos em delineamento inteiramente casualizado, com cinco tratamentos e seis repetições de 20 aves. Em cada fase, os tratamentos consistiram de níveis crescentes de lisina digestível nas rações. Os dados de conversão alimentar foram submetidos à análise de variância e posteriormente a análises de regressão utilizando-se um modelo não-linear exponencial. Com base nas equações exponenciais ajustadas para cada fase de criação, foram calculadas a variação do custo com alimentação e a margem por kg de ganho de peso. Em cada fase, foi considerado nível ótimo de lisina digestível aquele capaz de proporcionar o menor custo com alimentação e a maior margem por kg de ganho de peso. Considerando o preço da L-lisina $\mathrm{HCl}$ (78,5\%) de R\$ 11,78, os níveis ótimos de lisina digestível nas rações de crescimento e terminação são de 1,150 e 0,980\%, respectivamente. Entretanto, se o preço desta fonte cristalina aumentar para R\$ 14,13, os níveis ótimos nas rações reduzirão para 1,11 e 0,950\%, respectivamente.

Palavras-chave: aminoácidos, avicultura, desempenho, rentabilidade, viabilidade econômica

\section{Lysine levels in diets of broilers determined based on economic approach}

\begin{abstract}
The objective of this study was to estimate the optimum digestible lysine levels in diets for broilers on growing (22 to 35 days) and finishing (35 to 42 days) phases, based on economic analysis of food. Two experiments were conducted, each one using 600 Cobb 500 male broilers, distributed in a completely randomized design with five treatments and six replicates of 20 birds. Treatments consisted of increasing digestible lysine levels in the diet. The feed:gain relation data were submitted to ANOVA and to regression analyses using an exponential model. Based on adjusted equations for each phase, the change in food cost and margin per $\mathrm{kg}$ of weight gain was calculated. At each phase the optimum lysine level was considered as one to provide the lowest food cost and higher margin per kg of weight gain. Considering the price of L-lysine $\mathrm{HCl}(78.5 \%)$ of $\mathrm{R} \$ 11.78$, the optimum lysine levels in diets of growing and finishing poultry were estimated at 1.150 and $0.980 \%$ respectively. However, if the price of this source rises to $\mathrm{R} \$ 14.13$, the optimum lysine levels in diets will be reduced to 1.11 and $0.950 \%$ respectively.
\end{abstract}

Key Words: amino acids, economic viability, performance, poultry science, profitability

\section{Introdução}

O principal objetivo da indústria avícola moderna é a rentabilidade, que pode ser definida como o retorno financeiro obtido a partir do capital empregado. Contudo, os estudos tradicionalmente conduzidos para definir as exigências de aminoácidos de frangos de corte não consideram variáveis econômicas. Baseiam-se apenas na descrição quantitativa de variáveis produtivas ou indicadores metabólicos em resposta ao aumento da concentração desses nutrientes nas rações, sendo a exigência correspondente à concentração capaz de maximizar ou otimizar determinada resposta (Owens \& Pettigrew, 1989; Sakomura \& Rostagno, 2007).

De acordo com Pack et al. (2003), em estudos para quantificar as exigências de nutrientes essenciais, incluindo 
aminoácidos, deveriam ser consideradas variáveis de relevância econômica nas análises, pois as concentrações de aminoácidos que proporcionam máxima eficiência produtiva, na maioria das vezes, não coincidem com aquelas necessárias para alcançar a máxima lucratividade. Esses autores comentam que o termo "exigência", considerando como concentração fixa de aminoácidos nas rações, é empregado indevidamente, uma vez que existem diversos critérios de respostas, como ganho de peso, conversão alimentar, rendimento de carcaça ou peito, entre outros, que podem ser utilizados.

Outro fator que corrobora os relatos de Pack et al. (2003) é a variação individual existente em uma população de frangos de corte, uma vez que, ao definir uma concentração fixa visando atender às necessidades de um lote, existe a possibilidade de sobrefornecimento para aqueles indivíduos com menor potencial de resposta e subfornecimento de nutrientes para aqueles indivíduos com maior potencial de resposta. Diante disso, Sakomura \& Rostagno (2007) relataram que o mais importante é entender como uma população ou lote de animais responde ao acréscimo da concentração de um aminoácido na ração, identificando-se a região crítica das curvas-resposta, o que possibilita a associação de variáveis econômicas para definição dos níveis ótimos de aminoácidos nas rações.

Modelos de regressão não-linear da família exponencial têm sido recomendados por diversos autores (Pack, 1996; Pack et al., 2003; Lemme, 2005; Sakomura \& Rostagno, 2007; Siqueira et al., 2009) por obedecerem a "lei dos mínimos retornos", pela qual o incremento no desempenho decresce à medida que doses crescentes de um nutriente limitante são fornecidas, possibilitando a descrição detalhada do desempenho em resposta ao acréscimo de nutrientes nas rações, o que, em situações práticas, pode ser de grande utilidade quando o objetivo é determinar a concentração adequada de aminoácidos nas rações para proporcionar a máxima lucratividade.

Neste estudo objetivou-se estimar os níveis ótimos de lisina digestível em rações para frangos de corte em crescimento (22 a 35 dias) e terminação (35 a 42 dias) com base na análise econômica da alimentação utilizando-se equações exponenciais ajustadas aos dados de conversão alimentar.

\section{Material e Métodos}

Foram conduzidos dois experimentos no Setor de Avicultura do Departamento de Zootecnia da Faculdade de Ciências Agrárias e Veterinárias-FCAV/UNESP, Jaboticabal - SP, entre junho de 2008 e setembro de 2008, para estimar os níveis ótimos de lisina digestível em rações para frangos de corte nas fases de crescimento (22 a 35 dias) e terminação (35 a 42 dias), com base em uma abordagem econômica.

Foram utilizados 600 frangos de corte machos, da linhagem Cobb 500, com peso inicial de 771,74 $\pm 0,85 \mathrm{~g}$ na fase de crescimento e 1.737,03 $\pm 4,89 \mathrm{~g}$ na fase de terminação, totalizando 1.200 aves. Até o início de cada experimento, as aves foram criadas recebendo rações formuladas para atender suas exigências descritas por Rostagno et al. (2005) para cada fase.

Em cada experimento (22 a 35 e 35 a 42 dias), as aves foram distribuídas em delineamento inteiramente casualizado, com cinco tratamentos (níveis de lisina digestível na ração) e 6 repetições, sendo cada unidade experimental composta por 20 aves. No início de cada experimento, as aves foram pesadas individualmente para constituir parcelas com pesos iniciais homogêneos.

Para cada fase foram formuladas rações basais compostas principalmente de milho e farelo de soja, para atender às exigências nutricionais das aves de acordo com as recomendações de Rostagno et al. (2005), exceto em lisina. A suplementação de L-lisina $\mathrm{HCl}(78,5 \%)$ nas rações basais, em substituição ao ácido L-glutâmico e ao amido de milho, possibilitou a obtenção de rações experimentais isoenergéticas e isonitrogênicas com níveis crescentes de lisina digestível. Para garantir que nenhum outro aminoácido se tornasse limitante nas rações, a suplementação foi feita com fontes cristalinas de modo que as relações aminoácidos:lisina excedessem em pelo menos quatro pontos percentuais a relação de proteína ideal recomendada por Rostagno et al. (2005), garantindo que a lisina fosse o primeiro limitante (Tabelas 1 e 2).

Inicialmente, as pressuposições de normalidade e homocedasticidade foram testadas e atendidas. Em seguida, os dados de conversão alimentar de cada fase foram submetidos a análises de variância de acordo com o modelo estatístico: $\mathrm{CA}_{\mathrm{ij}}=\mu+\mathrm{Lys}_{\mathrm{i}}+\mathrm{e}_{\mathrm{ij}}$; em que $\mathrm{CA}_{\mathrm{ij}}=$ conversão alimentar observada no i-ésimo nível de lisina digestível na j-ésima repetição; $\mu=$ efeito da média geral; $\mathrm{Lys}_{\mathrm{i}}=$ efeito do i-ésimo nível de lisina digestível da ração; e $\mathrm{e}_{\mathrm{ij}}=$ erro experimental.

Posteriormente, os dados foram submetidos às análises de regressão considerando a conversão alimentar como variável dependente e o nível de lisina da ração como variável independente de acordo com o modelo exponencial descrito por Gahl et al. (1994) e Rodehutscord \& Pack (1999): $\mathrm{CA}_{\mathrm{i}}=\mathrm{A}+\mathrm{B} *\left(1-{ }^{e-\mathrm{C}(\text { Lysi-D })}\right)+\mathrm{e}_{\mathrm{i}}$; em que $\mathrm{CA}_{\mathrm{i}}=$ conversão alimentar estimada para o i-ésimo nível de lisina digestível na ração, Lys $_{i}=$ nível de lisina digestível da ração, A = resposta de conversão alimentar estimada para a ração 
Tabela 1 - Rações formuladas com níveis crescentes de lisina digestível para frangos de corte Cobb 500 na fase de crescimento (22 a 35 dias $)^{1}$

\begin{tabular}{|c|c|c|c|c|c|c|c|c|c|c|}
\hline \multirow[t]{2}{*}{ Ingrediente (\%) } & \multicolumn{10}{|c|}{ Nível de lisina digestível (\%) } \\
\hline & 0,786 & & 0,872 & & 0,958 & & 1,044 & & 1,130 & \\
\hline Milho & 65,838 & & 65,838 & & 65,838 & & 65,838 & & 65,838 & \\
\hline Farelo de soja & 25,726 & & 25,726 & & 25,726 & & 25,726 & & 25,726 & \\
\hline Fosfato bicálcico & 1,687 & & 1,687 & & 1,687 & & 1,687 & & 1,687 & \\
\hline Calcário calcítico & 0,857 & & 0,857 & & 0,857 & & 0,857 & & 0,857 & \\
\hline Óleo de soja & 2,431 & & 2,431 & & 2,431 & & 2,431 & & 2,431 & \\
\hline Cloreto de sódio & 0,470 & & 0,470 & & 0,470 & & 0,470 & & 0,470 & \\
\hline Sup. mineral ${ }^{2}$ & 0,050 & & 0,050 & & 0,050 & & 0,050 & & 0,050 & \\
\hline Sup. vitamínico ${ }^{3}$ & 0,025 & & 0,025 & & 0,025 & & 0,025 & & 0,025 & \\
\hline Cloreto de colina (70\%) & 0,070 & & 0,070 & & 0,070 & & 0,070 & & 0,070 & \\
\hline Anticoccidiano $^{4}$ & 0,050 & & 0,050 & & 0,050 & & 0,050 & & 0,050 & \\
\hline L-lisina $\mathrm{HCl}(78,5 \%)$ & - & & 0,110 & & 0,219 & & 0,329 & & 0,437 & \\
\hline DL-metionina (99\%) & 0,096 & & 0,161 & & 0,226 & & 0,292 & & 0,358 & \\
\hline L-treonina (99\%) & - & & 0,017 & & 0,078 & & 0,138 & & 0,197 & \\
\hline L-valina $(99 \%)$ & - & & - & & 0,047 & & 0,116 & & 0,185 & \\
\hline L-isoleucina (99\%) & - & & - & & 0,011 & & 0,072 & & 0,133 & \\
\hline L-triptofano (99\%) & - & & - & & 0,010 & & 0,027 & & 0,044 & \\
\hline L-arginina (99\%) & - & & - & & - & & 0,077 & & 0,170 & \\
\hline Ácido L-glutâmico (99\%) & 2,600 & & 2,342 & & 1,931 & & 1,167 & & 0,358 & \\
\hline Amido de milho & 0,100 & & 0,167 & & 0,273 & & 0,579 & & 0,920 & \\
\hline Total & 100,00 & & 100,00 & & 100,00 & & 100,00 & & 100,00 & \\
\hline Aminoácidos digestíveis (\%) & & aa/Lys & & aa/Lys & & aa/Lys & & aa/Lys & & aa/Lys \\
\hline Lisina $(100)^{5}$ & 0,786 & 100 & 0,872 & 100 & 0,958 & 100 & 1,044 & 100 & 1,130 & 100 \\
\hline Metionina + cistina (72) & 0,596 & 76 & 0,660 & 76 & 0,724 & 76 & 0,789 & 76 & 0,853 & 76 \\
\hline Metionina (40) & 0,348 & 44 & 0,412 & 47 & 0,476 & 50 & 0,541 & 52 & 0,605 & 58 \\
\hline Treonina (65) & 0,582 & 74 & 0,598 & 69 & 0,657 & 69 & 0,715 & 69 & 0,773 & 69 \\
\hline Valina (77) & 0,727 & 92 & 0,727 & 83 & 0,773 & 81 & 0,841 & 81 & 0,910 & 81 \\
\hline Isoleucina (67) & 0,665 & 85 & 0,665 & 76 & 0,676 & 71 & 0,736 & 71 & 0,796 & 71 \\
\hline Triptofano (17) & 0,184 & 23 & 0,184 & 21 & 0,193 & 20 & 0,210 & 20 & 0,227 & 20 \\
\hline Arginina (105) & 1,060 & 135 & 1,060 & 122 & 1,060 & 111 & 1,136 & 109 & 1,228 & 109 \\
\hline Leucina (109) & 1,467 & 187 & 1,467 & 168 & 1,467 & 153 & 1,467 & 141 & 1,467 & 130 \\
\hline Fenilalanina (63) & 0,792 & 101 & 0,792 & 91 & 0,792 & 83 & 0,792 & 76 & 0,792 & 70 \\
\hline Glicina + serina $(140)^{6}$ & 1,619 & 188 & 1,619 & 171 & 1,619 & 156 & 1,619 & 144 & 1,619 & 134 \\
\hline
\end{tabular}

${ }^{1}$ Composição calculada: energia metabolizável = $3.100 \mathrm{kcal} / \mathrm{kg}$; proteína bruta = 19,37\%; fósforo disponível = 0,411\%; cálcio = 0,824\%; sódio = 0,205\%; fibra bruta = $2,53 \%$.

2 Conteúdo/kg: $\mathrm{Mn}=150.000 \mathrm{mg} ; \mathrm{Fe}=100.000 \mathrm{mg} ; \mathrm{Zn}=100.000 \mathrm{mg} ; \mathrm{Cu}=16.000 \mathrm{mg} ; \mathrm{I}=1.500 \mathrm{mg} ;$ veículo qsp = $1.000 \mathrm{~g}$.

${ }^{3}$ Conteúdo $/ \mathrm{kg}$ : ácido fólico $=700 \mathrm{mg}$; ácido pantotênico $=13.000 \mathrm{mg}$; niacina $=35.000 \mathrm{mg} ;$ vit. B1 = $1.600 \mathrm{mg}, \mathrm{vit}$. B12 = $10.000 \mathrm{mg}$, vit. B2 = 5.000 mg, vti. B6 = $2.600 \mathrm{mg}$, vit. D3 = 1.500.000 UI, vit. E = $12.000 \mathrm{mg}$; vit. K3 = $1.500 \mathrm{mg}$; Se = $300 \mathrm{mg}$; antioxidante = $500 \mathrm{mg} ;$ veículo qsp = $1.000 \mathrm{~g}$.

4 Salinomicina sódica $=60 \mathrm{ppm}$.

5 Relações na proteína ideal (aa/Lys) recomendadas por Rostagno et al. (2005).

${ }^{6}$ Valores e relações definidos com base em aminoácidos totais (conforme Tabela 2).

contendo o nível mais baixo de lisina digestível (basal), $\mathrm{B}$ = diferença estimada entre a mínima e a máxima resposta obtida com a adição de lisina, $\mathrm{C}$ = coeficiente de inclinação da curva, $\mathrm{D}$ = nível de lisina da ração basal, $e$ = base do logaritmo neperiano (2,718282), $\mathrm{e}_{\mathrm{i}}=$ erro ou desvio associado à distância entre o valor observado e o valor estimado da conversão alimentar.

Com base nas equações exponenciais ajustadas para cada fase de criação, foi calculada a variação do custo com alimentação por kg de ganho de peso, conforme descrito por Pack et al. (2003). A variação no custo da alimentação $(\Delta \mathrm{R} \$)$, proporcionada pela adição de níveis crescentes de lisina digestível, foi obtida para os intervalos testados pela expressão: $\Delta \mathrm{R} \$=(\mathrm{CDB}+((\mathrm{CUAA}-\mathrm{CUDB}) * \mathrm{NSAA}))$; em que: $\mathrm{CDB}$ = custo do $\mathrm{kg}$ da dieta basal (sem adição de L-lisina $\mathrm{HCl})(\mathrm{R} \$)$; CUAA = custo por unidade da lisina digestível suplementar $(\mathrm{R} \$)$; CUDB = custo unitário da dieta basal (R\$) e NSAA = acréscimo na concentração de lisina digestível (\%) proporcionado pela suplementação de L-lisina $\mathrm{HCl}(78,5 \%)$ na dieta basal $\left(\mathrm{Lys}_{\mathrm{i}}-\mathrm{D}\right)$. Multiplicando-se os valores de conversão alimentar (CR/GP) estimados pela equação exponencial para cada nível de lisina digestível suplementar, pelo custo do alimento obtido para cada nível de suplementação, obteve-se a variação do custo com alimentação/kg de GP ( $\Delta$ R \$/kgGP) nos intervalos testados.

A margem por kg de frango para cada nível de suplementação de lisina digestível nas rações foi calculada pela expressão: $\mathrm{M}=\mathrm{PVF}-(\mathrm{R}$ /kgGP); em que $\mathrm{M}$ = margem por kg de frango $(\mathrm{R} \$) ; \mathrm{PVF}=$ preço de venda do frango vivo (R\$/kg), e (R\$/kgGP) é o custo com alimentação por kg de ganho de peso vivo dos frangos.

Em cada fase, os níveis ótimos de lisina nas rações, obtidos com base na abordagem econômica, foram definidos 
Tabela 2 - Rações formuladas com níveis crescentes de lisina digestível para frangos de corte Cobb 500 na fase de terminação (35 a 42 dias $)^{1}$

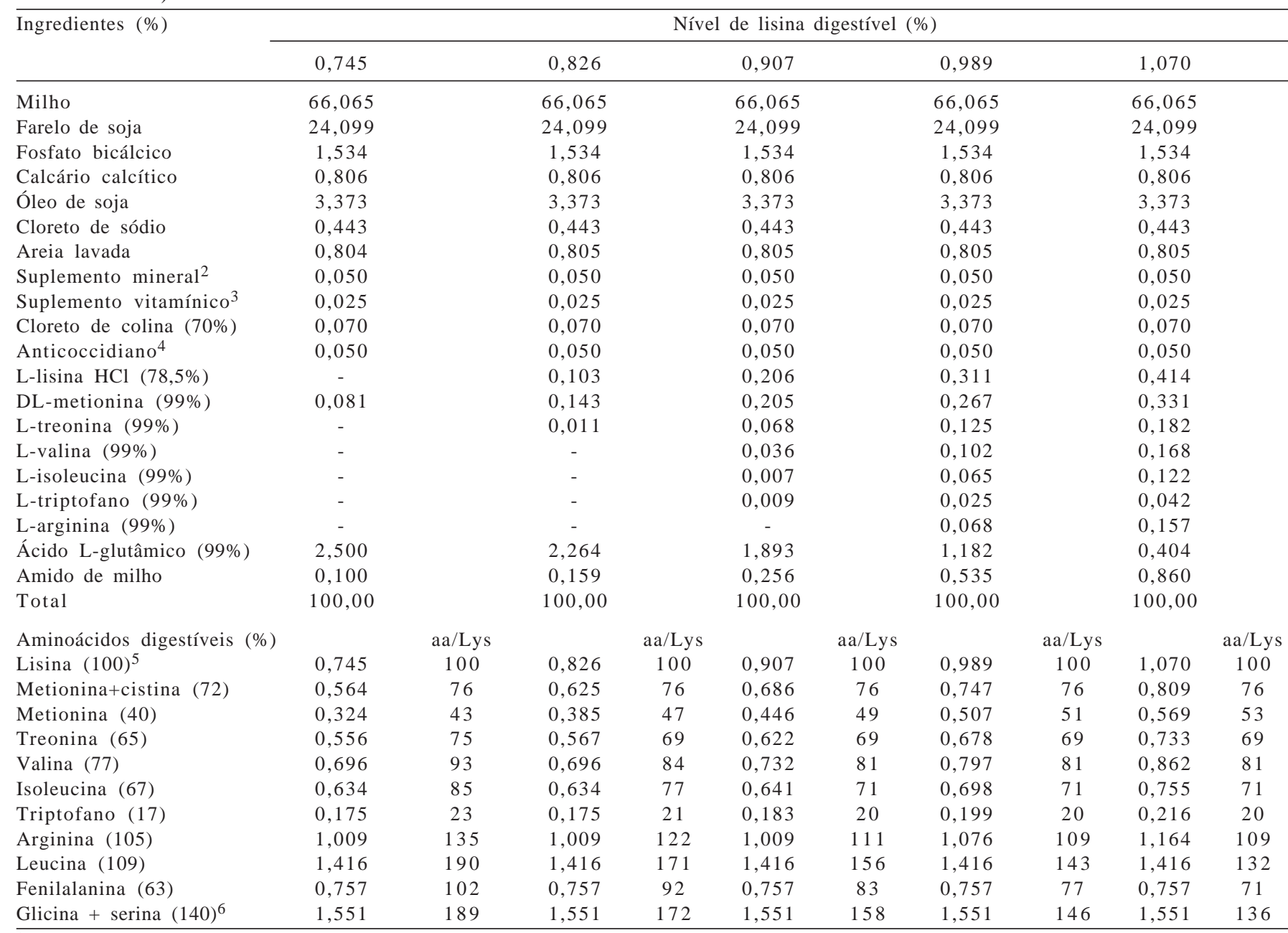

${ }^{1}$ Composição calculada: energia metabolizável = $3.150 \mathrm{kcal} / \mathrm{kg}$; proteína bruta = 18,57\%; fósforo disponível = 0,380\%; cálcio = 0,763\%; sódio = 0,194\%.

${ }^{2}$ Conteúdo/kg: $\mathrm{Mn}=150.000 \mathrm{mg} ; \mathrm{Fe}=100.000 \mathrm{mg} ; \mathrm{Zn}=100.000 \mathrm{mg} ; \mathrm{Cu}=16.000 \mathrm{mg} ; \mathrm{I}=1.500 \mathrm{mg} ;$ veículo qsp = $1.000 \mathrm{~g}$.

3 Conteúdo/kg: ácido fólico = $700 \mathrm{mg}$; ácido pantotênico = $13.000 \mathrm{mg}$; niacina = $35.000 \mathrm{mg}$; vit. B1 = 1.600 mg; vit. B12 = $10.000 \mathrm{mg} ;$ vit. B2 = $5.000 \mathrm{mg} ;$ vit. B6 = 2.600 mg;

vit. D3 = 1.500.000 UI; vit. E = $12.000 \mathrm{mg}$; vit. K3 = $1.500 \mathrm{mg}$; Se = $300 \mathrm{mg}$; antioxidante = $500 \mathrm{mg}$; veículo qsp = 1000 g.

4 Salinomicina sódica $=60 \mathrm{ppm}$.

5 Relações na proteína ideal (aa/Lys) recomendadas por Rostagno et al. (2005).

${ }^{6}$ Valores e relações definidos com base em aminoácidos totais.

como sendo aqueles que proporcionaram os menores custos com alimentação por kg de ganho de peso e a maior margem.

As análises estatísticas foram realizadas utilizando-se o programa computacional SAS 9.0, por meio dos procedimentos GLM para análise de variância e NLIN (Gauss-Newton) para o ajuste do modelo exponencial (SAS, 1985).

\section{Resultados e Discussão}

Experimentos dose-resposta delineados para definir as exigências nutricionais de frangos de corte têm sido tradicionalmente analisados por meio de modelos de regressão de natureza linear, entre eles, o polinomial quadrático e o Linear Response Plateau (LRP) ou Broken Line.
Segundo Oviedo-Rondón et al. (2002), os modelos de natureza linear são amplamente utilizados na avicultura por serem facilmente implementados nas análises estatísticas. Pack et al. (2003) salientaram que a popularidade desses modelos ocorre pela sua interpretação simplificada das respostas biológicas, possibilitando a determinação matemática de um único valor do nutriente testado, considerado como sendo a exigência.

Entretanto, este tipo de determinação não considera aspectos econômicos e sabe-se que o nível ótimo determinado com base no desempenho na maioria das vezes não coincide com o ótimo econômico, e o principal objetivo do produtor é a lucratividade. Com base nisso, diversos autores (Pack et al., 2003; Lemme, 2005; Sakomura \& Rostagno, 2007; Siqueira et al., 2009) têm recomendado o uso de modelos de 
regressão não-linear, por permitirem a descrição detalhada da curva-resposta, possibilitando a associação de variáveis econômicas para determinação dos níveis ótimos de nutrientes nas rações.

Neste estudo, os níveis de lisina digestível das rações influenciaram $(\mathrm{P}<0,001)$ a conversão alimentar dos frangos de corte Cobb 500 nas fases de crescimento (22 a 35 dias) e terminação (35 a 42 dias), que melhorou com o aumento da concentração de lisina em ambas as fases. Esse resultado era esperado, tendo em vista a essencialidade desse aminoácido para frangos de corte.

O modelo de regressão exponencial foi adequado para descrever as respostas de conversão alimentar das aves em função dos níveis de lisina digestível das rações em ambas as fases, proporcionando excelentes ajustes do ponto de vista estatístico (Figuras 1 e 2).

Para os cálculos do nível ótimo de lisina digestível com base na viabilidade econômica, o custo da ração basal (sem adição de L-lisina $\mathrm{HCl}$ ) para a fase de crescimento foi considerado como R \$ 0,76/kg e, para a fase de terminação, como R \$ 0,74/kg, admitindo-se que na fase de crescimento as rações têm maior densidade de nutrientes em relação à fase de terminação, sendo, portanto, mais caras.

Para cada fase, os cálculos foram realizados considerando o custo da L-lisina $\mathrm{HCl}(78,5 \%)$ como $\mathrm{R} \$ 11,78 / \mathrm{kg}$ ou $\mathrm{R} \$ 14,13 / \mathrm{kg}$ (+20\%), assumindo a digestibilidade verdadeira dessa fonte cristalina como 100\% (Han \& Baker, 1994). Desse modo, o custo da lisina digestível proporcionado pela adição de L-lisina $\mathrm{HCl}(78,5 \%)$ foi de R\$15,00/kg $(11,78 / 0,785=15,00)$ ou $\mathrm{R} \$ 18,00 / \mathrm{kg}(14,13 / 0,785=18,00)$, gerando estimativas distintas do nível ótimo em cada uma dessas situações.

$\mathrm{Na}$ fase de crescimento, assumindo o custo da lisina digestível de $\mathrm{R} \$ 15,00 / \mathrm{kg}$ (L-lisina $\mathrm{HCl}=\mathrm{R} \$ 11,78$ ), o menor custo com alimentação por kg de ganho de peso foi de aproximadamente $\mathrm{R} \$ 1,312(\mathrm{R} \$ / \mathrm{kgGP}=(0,76+(0,15-$ $0,0076) * 0,37) * 1,6147=1,312)$. Assumindo o preço de venda do frango vivo como R $\$ 1,65 / \mathrm{kg}$, a margem por kg de frango produzido foi de $\mathrm{R} \$ 0,338(\mathrm{M}=1,65-1,312=0,338)$, correspondendo ao nível de 1,150\% de lisina digestível na ração, capaz de proporcionar o menor custo com alimentação e a maior margem, situando-se acima do maior nível de lisina

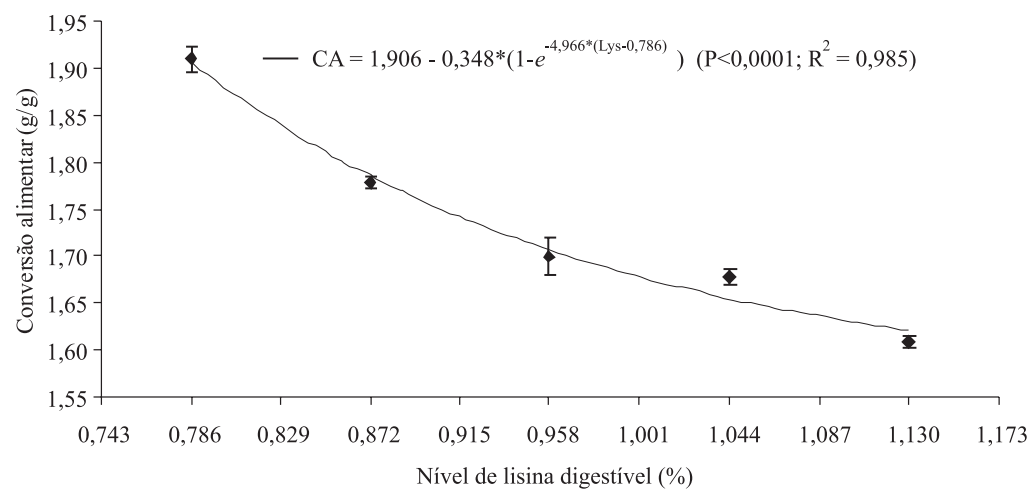

Figura 1 - Modelo exponencial ajustado aos dados de conversão alimentar (CA) de frangos de corte Cobb 500 na fase de crescimento (22 a 35 dias).

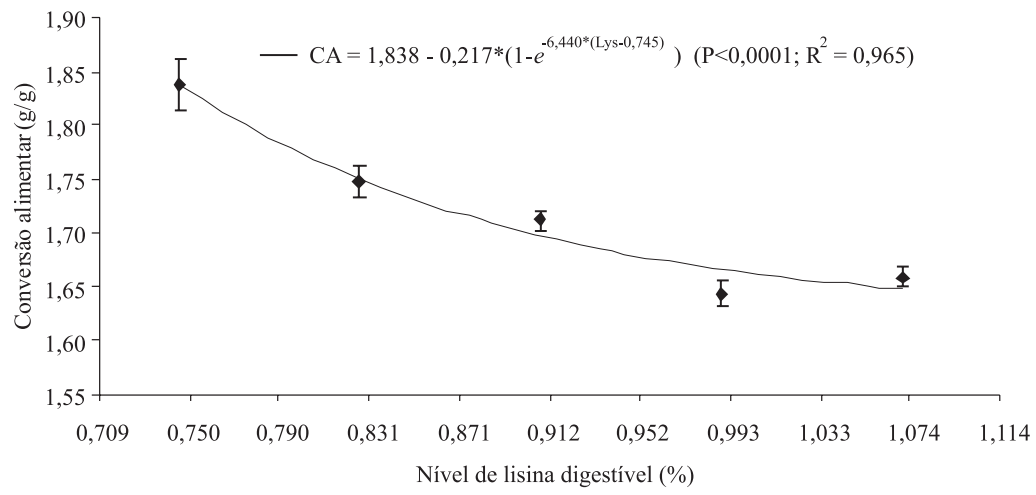

Figura 2 - Modelo exponencial ajustado aos dados de conversão alimentar (CA) de frangos de corte Cobb 500 na fase de terminação (35 a 42 dias). 
testado (1,130\%). Entretanto, tendo em vista as oscilações no preço de mercado dos insumos utilizados na nutrição avícola, se o preço da lisina digestível proveniente da suplementação de L-lisina $\mathrm{HCl}$ aumentar para $\mathrm{R} \$ 18,00$ ( L-lisina $\mathrm{HCl}=\mathrm{R} \$ 14,13$ ), o menor custo com alimentação por kg de ganho de peso passará a ser de R \$1,329 (R \$/kgGP = $(0,76+(0,18-0,0076) * 0,33) * 1,6272=1,329)$, resultando em uma margem por kg de frango produzido de $\mathrm{R} \$ 0,321(\mathrm{M}=$ $1,65-1,329=0,321$ ), menor que na situação anterior. Neste caso, o nível ótimo de lisina digestível na ração reduzirá para 1,110\% (Figura 3).

Na fase de terminação, os resultados obedeceram à mesma tendência, e o menor custo com alimentação por kg de ganho de peso calculado em aproximadamente $\mathrm{R} \$ 1,292$ $(\mathrm{R} \$ / \mathrm{kgGP}=(0,74+(0,15-0,0074) * 0,24) * 1,6688=1,292)$ quando o preço da lisina foi considerado $\mathrm{R} \$ 15,00 / \mathrm{kg}$, resultando em margem por $\mathrm{kg}$ de frango produzido de $\mathrm{R} \$ 0,358,(\mathrm{M}=1,65-1,292=0,358)$, assumindo o mesmo preço de venda do frango vivo (R\$ 1,65/kg). Neste caso, o nível ótimo de lisina digestível na ração correspondeu a 0,980\%. Por outro lado, se o preço da lisina digestível aumentar para $\mathrm{R} \$ 18,00$, o menor custo com alimentação por kg de ganho de peso passará a ser de R \$1,303 (R \$/kgGP =
$(0,74+(0,18-0,0074) * 0,21) * 1,6790=1,303)$, resultando em margem por kg de frango produzido de $\mathrm{R} \$ 0,347(\mathrm{M}=1,65$ -1,303 = 0,347). Assim, o nível ótimo de lisina digestível na ração capaz de proporcionar o menor custo com alimentação e a maior margem por kg de frango produzido reduzirá para 0,950\% (Figura 4).

A utilização do modelo exponencial permite identificar o ponto da curva-resposta em que o aumento relativo no custo da alimentação proporcionado pela suplementação de lisina digestível se iguala à melhora relativa no desempenho. Assim, se o preço da L-lisina $\mathrm{HCl}(78,5 \%)$ aumentar, o custo com a alimentação para cada nível de suplementação de lisina digestível também aumentará, sendo coerente que o nível capaz de proporcionar o menor custo com alimentação seja inferior, como ilustrado neste estudo.

Para as condições nas quais os cálculos foram realizados, observaram-se maiores restrições econômicas para a fase de terminação em relação à fase de crescimento, visto que o custo com alimentação passou a aumentar e a margem a diminuir a partir de níveis relativamente inferiores. Esse comportamento está diretamente relacionado ao maior potencial de resposta das aves na fase de crescimento $(B=0,348)$ em relação àquelas em terminação $(B=0,217)$,

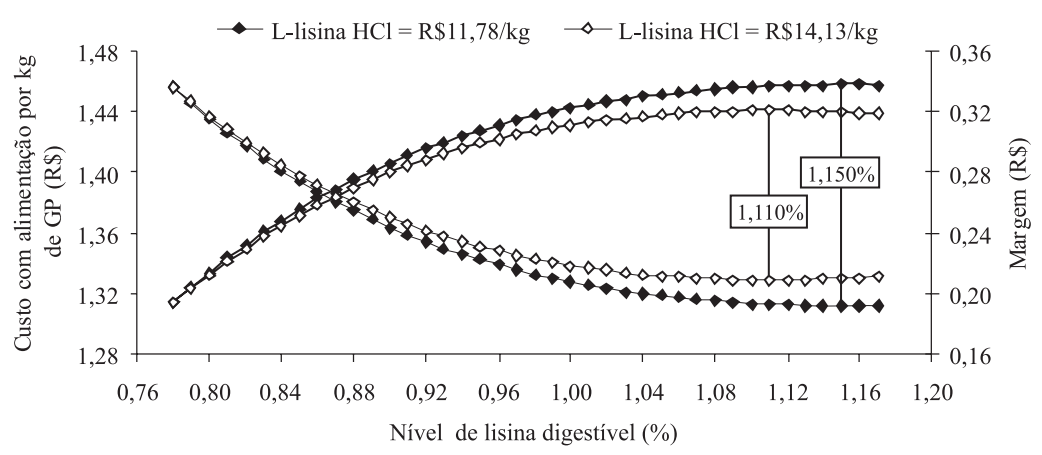

Figura 3 - Variação do custo com alimentação e da margem por kg de frango produzido de acordo com o nível de lisina digestível da ração para frangos de corte Cobb 500 em crescimento (22 aos 35 dias).

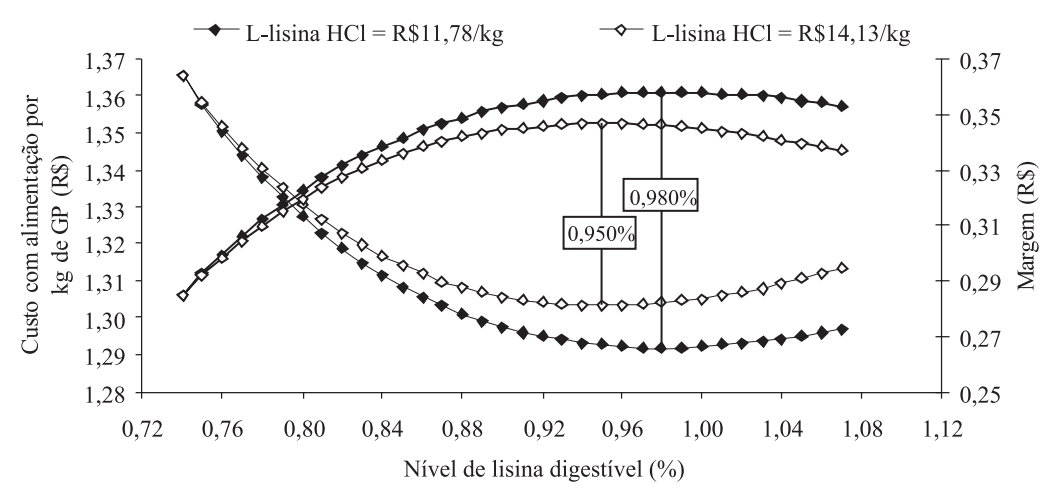

Figura 4 - Variação do custo com alimentação e da margem por kg de frango produzido de acordo com o nível de lisina digestível da ração para frangos de corte Cobb 500 em terminação (35 aos 42 dias). 
e consequentemente, com os valores dos parâmetros estimados para as equações de cada fase, especialmente o parâmetro C (coeficiente de inclinação da curva), que ditam o grau de curvatura das respostas.

Valores relativamente maiores do parâmetro C, como na fase de terminação $(C=6,440)$, geram curvas-resposta cuja região crítica (região onde a probabilidade de resposta diminui) encontra-se em limites relativamente estreitos, não havendo maiores benefícios no desempenho com a suplementação de níveis elevados. Por outro lado, quando são estimados valores relativamente menores de $C$, no caso da fase de crescimento $(C=4,996)$, as respostas encontram-se melhor distribuídas, proporcionando reduções no custo por kg de ganho com a suplementação de níveis mais elevados de lisina digestível.

Ferreira et al. (2009) utilizaram o modelo exponencial para definir o nível ótimo econômico de metionina+cistina em rações de frangos de corte em terminação e os resultados podem confirmar essas afirmações. O parâmetro C estimado por esses autores foi extremamente elevado $(22,36)$, resultando em uma região crítica da curva-resposta bem delimitada, de modo que reduções acentuadas no custo com alimentação ocorreram somente com dietas extremamente deficientes.

Siqueira et al. (2009) utilizaram abordagem semelhante para definir o nível ótimo econômico de lisina digestível em rações para frangos de corte da linhagem ISA Label. Os autores estimaram o parâmetro $\mathrm{C}$ do modelo exponencial em 13,88, resultando em níveis econômicos inferiores aos níveis adequados para melhorar o desempenho das aves. Esses resultados confirmam a influência do valor do parâmetro C do modelo exponencial na determinação do nível ótimo econômico de nutrientes com base nessa abordagem.

Vale mencionar que o modelo de regressão exponencial baseia-se no princípio de que o aumento na resposta animal diminui à medida que se aproxima do máximo desempenho (Sakomura \& Rostagno, 2007), não predizendo o efeito causado pelo excesso do nutriente avaliado. Assim, para sua correta utilização, é necessário que haja número suficiente de níveis do nutriente avaliado nas rações e que estes partam de uma condição de grande deficiência até uma condição a partir da qual não haja grandes aumentos na resposta (Pack et al., 2003).

A abordagem econômica utilizada neste estudo considerou apenas os custos com alimentação, não envolvendo outros componentes do custo de produção. Entretanto, sabe-se que, na avicultura de corte, o custo com alimentação corresponde à aproximadamente $70 \%$ do custo total de produção (Nascimento et al., 2009; Rizzo et al., 2010). Com base nisso, a utilização do modelo exponencial para definição dos níveis adequados de nutrientes nas rações de frangos de corte pode ser uma ferramenta de grande importância para auxiliar nas decisões em situações práticas, uma vez que os preços dos insumos utilizados na nutrição animal estão sujeitos a frequentes oscilações de mercado.

\section{Conclusões}

Considerando o preço da L-lisina $\mathrm{HCl}(78,5 \%)$ de R \$11,78, os níveis ótimos de lisina digestível nas rações para crescimento e terminação são de 1,150 e 0,980\%, respectivamente, pois proporcionam as maiores margens. Entretanto, se o preço dessa fonte cristalina aumentar para R \$ 14,13, os níveis ótimos nas rações para crescimento e terminação reduzirão para 1,110 e 0,950\%, respectivamente, havendo consequentemente redução na margem. A utilização do modelo exponencial pode ser valiosa em decisões práticas, tendo em vista que os preços dos insumos utilizados na nutrição animal estão sujeitos a frequentes oscilações de mercado.

\section{Agradecimentos}

À Fundação de Amparo à Pesquisa do Estado de São Paulo (FAPESP) pelo financiamento da pesquisa.

Ao Conselho Nacional de Desenvolvimento Científico e Tecnológico (CNPQ) pelo fornecimento de bolsas de estudo e pesquisa.

À Ajinomoto Biolatina Indústria e Comércio Ltda. pela doação dos aminoácidos.

\section{Referências}

FERREIRA, N.T.; SAKOMURA, N.K.; SIQUEIRA, J.C. et al. Níveis de metionina+cistina para frangos de corte determinados com base na análise econômica da alimentação. In: REUNIÃO ANUAL DA SOCIEDADE BRASILEIRA DE ZOOTECNIA, 46. 2009, Maringá. Anais... Maringá: UEM 2009. (CD-ROM).

GAHL, M.J.; CRENSHAW, T.D.; BENEVENGA, N.J. Diminishing returns in weight, nitrogen, and lysine gain of pigs fed six levels of lysine from three supplemental sources. Journal of Animal Science, v.72, p.3177-3187, 1994.

HAN, Y.; BAKER, D.H. Digestible lysine requirement of male and female broiler chicks during the period three to six weeks post hatching. Poultry Science, v.73, p.1739-1745, 1994.

LEMME, A. Optimum dietary amino acid level for broiler chicken. In: SIMPÓSIO INTERNACIONAL SOBRE EXIGENCIAS NUTRICIONAIS DE AVES E SUÍNOS, 2., 2005, Viçosa, MG. Anais. Viçosa, MG: UFV, 2005. p.117-144.

NASCIMENTO, D.C.N.; SAKOMURA, N.K.; SIQUEIRA, J.C. et al. Exigências de metionina + cistina digestível para aves de corte ISA Label criadas em semiconfinamento. Revista Brasileira de Zootecnia, v.38, n.5, p.869-878, 2009.

OVIEDO-RONDÓN, E.O.; MURAKAMI, A.E.; SAKAGUTI, E.S. Modelagem computacional para produção e pesquisa em avicultura. Revista Brasileira de Ciência Avícola, v.4, p.199-207, 2002. 
OWENS, F.N.; PETTIGREW, J.E. Subdividing amino acid requirements into portions for maintenance and growth. In: FRIEDMAN, M. (Ed.). Absorption and utilization of amino acids. Boca Raton: CRC Press, 1989. v.1, p.15-30.

PACK, M.; HOEHLER, D.; LEMME, A. Economic assessment of amino acid responses in growing poultry. In: D'MELLO, J.P.F. (Eds.) Amino acids in animal nutrition. Cambridge: CABI Publishing, 2003. p.459-483.

RIZZO, P.V.; MENTEN, J.F.M.; RACANICCI, A.M.C. et al. Extratos vegetais em dietas para frangos de corte. Revista Brasileira de Zootecnia, v.39, n.4, p.801-807, 2010.

RODEHUTSCORD, M.; PACK, M. Estimates of essential amino acid requirements from dose-response studies with rainbow trout and broiler chicken: effect of mathematical model. Archives of Animal Nutrition, v.52, p.223-244, 1999. ROSTAGNO, H.S.; ALBINO, L.F.T.; DONZELE, J.L. et al. Tabelas brasileiras para aves e suínos: composição de alimentos e exigências nutricionais. Viçosa, MG: Departamento de Zootecnia, 2005. 186p.

SAKOMURA, N.K.; ROSTAGNO, H.S. Métodos de pesquisa em nutrição de monogástricos. Jaboticabal: Funep, 2007. 283p. SIQUEIRA, J.C.; SAKOMURA, N.K.; NASCIMENTO, D.C.N. et al. Modelos matemáticos para estimar as exigências de lisina digestível para aves de corte ISA Label. Revista Brasileira de Zootecnia, v.38, n.9, p.1732-1737, 2009.

STATISTICAL ANALYSIS SYSTEM - SAS. SAS user's guide: statistics. 5.ed. Cary: SAS Institute, 1985. 956p. 